\title{
La adquisición de la estructura silábica: El caso del castellano limeño
}

Cecilia Ciccia Gabillo, Universidad de Lima (Perú); Oriele Montezuma del Castillo, Universidad de Lima (Perú), José Elías Ulloa, Universidad de Stony Brook, Nueva York (Estados Unidos); Gabriela Gabillo Ciccia, Colegio Roosevelt, Lima (Perú); Mery

Echenique Herrera, Instituto de Educación Especial "Santa Magdalena Sofía Barat",

Lima (Perú); Gissella Raffo Castro, Centro Learn and Play, Lima (Perú); Ana María Seminario Olórtigue, Universidad de Lima (Perú)

Recibido: 3 de febrero del 2009 / Aprobado: 10 de agosto del 2009

Esta investigación se centra en el estudio de las estructuras silábicas que muestran divergencias cuando se compara el habla del modelo adulto con el habla de niños limeños monolingües del castellano hablado en Lima. También busca determinar hasta qué edad subsisten dichas divergencias en el habla de los niños entre los dos años y medio y los cinco años y que no presentan aparentes limitaciones articulatorias, auditivas o intelectuales. Uno de los principales resultados de este trabajo es la identificación del momento en que se produce una disminución significativa en este tipo de divergencias, la mayoría de las cuales desaparecen entre los dos años y medio y los tres años y medio. Sin embargo, los casos de diptongación pueden subsistir hasta los cuatro años de edad.

desarrollo del lenguaje / estructura silábica / evaluación de procesos fonológicos

\section{The acquisition of syllable structure: The case study of the Spanish spoken in Lima}

This article focuses on which syllabic structure shows divergences when adult and child speech, of monolingual of the same city Spanish spoken in Lima, are compared. It also discusses the age until those divergences remain in the speech of children, between the ages of two and a half and five. No of the subjects present any articulatory, auditory or mental disability. The main result is the identification that between the ages of two and a half and three and a half, a significant decrease in most of the divergences related to syllable structure occurs. However, the cases of diphthongization could remain until the age of four years.

language development / phonological processes assessment / syllable structure

Correos electrónicos: Ccciccia@ulima.edu.pe; omontezu@correo.ulima.edu.pe; Jose.Elias-Ulloa@sunysb.edu; gabigabillo@terrra.com.pe; echenique_m@yahoo.es; gisellaraffo@hotmail.com; jladso@terra.com.pe 


\section{INTRODUCCIÓN}

La necesidad del lenguaje para el ser humano es tan grande que cuando surgen dificultades, estas repercuten en todas las áreas del desarrollo y si dichas dificultades persisten a lo largo de la vida escolar, llegan a ser una de las principales causas de los trastornos del aprendizaje.

Con el fin de contribuir tanto con la detección temprana de los trastornos específicos del lenguaje (TEL), así como también con los estudios de la adquisición fonológica y tomar las acciones preventivas y terapéuticas oportunas, este artículo se centra en la investigación de la adquisición de la sílaba en el castellano peruano. En particular, este estudio tiene como objetivo determinar qué patrones silábicos, presentes en el modelo adulto del castellano hablado en la ciudad de Lima, muestran divergencias cuando se compara con el habla infantil y hasta qué edad dichas divergencias subsisten en niños sin aparentes limitaciones articulatorias, auditivas o intelectuales.

El presente estudio complementa los hallazgos reportados en Ciccia et al. (2006). En dicho trabajo se identificó el rango de edades donde ocurre una disminución dramática de las divergencias entre el habla adulta del castellano peruano y la del niño con respecto a los rasgos fonológicos. Se encontró que este periodo crítico ocurre entre los dos años y medio y los tres años y medio. Tal hallazgo representa un punto de referencia para detectar posibles trastornos fonológicos. En contraste con el trabajo mencionado, el cual se enfoca en las divergencias relacionadas con los rasgos fonológicos, esta investigación toma como punto central de exploración la adquisición de la sílaba del castellano peruano y sus divergencias con respecto al modelo adulto.

El proceso de adquisición fonológica comienza desde el nacimiento con gritos y llantos que poco a poco van modificándose y al segundo mes aparecen vocalizaciones de las que saldrán los fonemas Lenneberg, prosigue con la emisión de los primeros sonidos y en el caso del castellano, continúa hasta cuando el niño produce consonantes vibrantes simples (por ejemplo, 'pero') y múltiples (por ejemplo, 'perro'). Entre los seis y los siete años es el periodo en que se logra dominar la fonología castellana (Acosta, 1999).

Aproximadamente a los dos años de edad se da el primer momento donde es posible empezar a comparar el habla adulta con el habla infantil, pues el niño ya produce muchas palabras. Por supuesto, a esta edad se observan muchísimas divergencias entre ambas producciones, que hacen el habla de los nenes sea abismalmente diferente a la del adulto y en consecuencia muy dificil de entender. No es raro encontrar niños de entre dos y tres años para quienes solo la madre o ambos padres 
pueden reconocer sus palabras mientras que otras personas - menos acostumbradas a tales producciones- dificilmente podrían saber a qué se refiere el niño. Sin embargo, aún con estas profundas divergencias en este periodo, ya es posible identificar la agrupación de sonidos lingüísticos en patrones silábicos y estos en palabras. Desafortunadamente, como se menciona en Aguado (1999), existen pocos estudios detallados sobre la producción del habla en niños de esa edad que nos permitan identificar indicios de posteriores trastornos fonológicos.

Trabajos como los de Acosta, Ramos y León (1998) y Bosch (1982, 1984, 2003) señalan la importancia del estudio fonológico de los patrones silábicos. El trabajo de Acosta, Ramos y León (1998) informa que en el habla de los niños de entre tres y siete años se presenta una gran cantidad de simplificaciones que afectan la estructura de la sílaba (por ejemplo, reducción de grupos consonánticos, reducción de diptongos y omisión de vibrantes), además de otras divergencias que afectan la estructura silábica (la semiconsonantización de vibrantes).

En forma independiente, Bosch (1982, 1984, 2003) llega a conclusiones similares. Su trabajo presenta un estudio detallado de los procesos de simplificación que afectan la estructura de la sílaba en el transcurso del desarrollo fonológico. La autora observa que tales procesos van desapareciendo ordenada y progresivamente, hasta que a la edad de seis años estos aparecen en un porcentaje tan ínfimo que su presencia se considera como nula en el desarrollo fonológico normal.

Hodson (1986), en trabajos sobre el problema del desarrollo del lenguaje y su diagnóstico, estudió las diferencias entre el habla inteligible y no inteligible tanto en inglés como en español, encontrando que las divergencias relacionadas con las estructuras silábicas (reducción de grupos consonánticos, omisiones de sílabas y elisión de segmentos) eran determinantes.

Los escolares que manifiestan dificultades fonológicas presentarán, durante la adolescencia y la adultez, desempeño debajo del nivel de los grupos de control en habla, lectura, deletreo y tareas de conciencia fonológica. Asimismo, aquellos que eliden sílabas en palabras multisilábicas parecen tener gran dificultad en el uso de palabras no acentuadas o funcionales, como el artículo (Lewis \& Freebaim, 1992, tomado de Flecher \& Winney, 1995, citado en Acosta \& Moreno, 1999).

La preferencia por la sílaba VCV y un repertorio consonántico restringido en niños de dos años de edad son dos características que indicarían la existencia de riesgo de retraso de lenguaje (Rescola \& Tatner, 1996, citado en Narbona, 2006). 


\section{MARCO TEÓRICO}

El lenguaje se compone, desde el punto de vista lingüístico, de tres dimensiones (Bloom \& Lahey, 1978): forma (fonología, morfología, sintaxis), uso (pragmática) y contenido (semántica). Nuestro estudio se centra en el componente fonológico y en particular se enmarca en el nivel de la sílaba.

Para lograr el objetivo de identificar las divergencias en la estructura silábica entre el modelo del habla adulta y el habla de los niños de 2,6 a cinco años vamos a utilizar, y en muchos casos adaptar a las necesidades de nuestra investigación, ciertos conceptos y herramientas que nos brinda la teoría fonológica moderna (Baertsch, 2002; Blevins, 1995; Bromberger \& Halle, 1989; Broselow, Chen \& Huffman, 1997; Cairns \& Feinstein, 1982; Halle, 2002; Halle \& Idsardi, 1995; J. Harris, 1983; J. W. Harris, 1989; Hironymous, 1999; Kenstowicz, 1994; Lee, 1989; Levelt, Schiller \& Levelt, 1999).
En esta sección abordaremos dos temas importantes que hacen explícitos nuestros supuestos teóricos. Primero, discutiremos qué entendemos por divergencia dentro del contexto de los estudios del lenguaje infantil y por qué preferimos este término al de proceso (fonológico) o error. La segunda parte de esta sección está dedicada a explicar el marco teórico que utilizaremos para estudiar los patrones silábicos.

\section{Divergencias}

Las divergencias se refieren a las diferencias que durante el tiempo de adquisición fonológica se observan cuando se comparan las producciones del habla adulta con las de los niños. Por ejemplo, mientras que en el modelo adulto (peruano) la palabra 'globo' se pronuncia [glo.bo], un niño de tres años que está adquiriendo el castellano podría pronunciarla como [go.bo]. Es decir, comparado con el modelo adulto, el niño ha simplificado el grupo consonante mediante la elisión de la consonante [1]. El punto en las transcripciones indica las divisiones silábicas.

Cuadro 1

Las producciones del adulto y de los niños

Producción del modelo adulto

glo.bo
Producción del niño que

está adquiriendo castellano

go.bo 
En nuestro estudio consideraremos la producción del modelo adulto como una forma ideal a la cual la producción del niño intenta alcanzar. Dependiendo del estadio de adquisición, la producción del habla del niño puede mostrar diferentes grados de divergencia con respecto a la producción del modelo adulto.

En los estudios fonológicos se distingue entre dos tipos de representaciones mentales: una más abstracta e ideal y otra que tiene más información sobre detalles fonéticos, la cual se aleja de la representación ideal. Estas son las representaciones fonológicas y las representaciones fonéticas, respectivamente.

Así, por ejemplo, la representación fonológica en castellano del sonido /b/ incluye ser oclusivo (i.e. el rasgo [-continuo]). Esto se puede comprobar al pronunciar la palabra 'barco.' Los labios se cierran completamente para hacer el sonido /b/y en consecuencia el aire deja de fluir a través de la boca. Lo interesante es que cuando el mismo sonido ocurre entre vocales ya no es oclusivo sino fricativo: $[\beta]$, porque el aire sale continuamente a través de la boca durante su articulación. Esto se puede observar al pronunciar la palabra 'cabo'. A pesar de ser fricativo entre vocales, en el nivel fonológico aún se le va a considerar oclusivo: $/ \mathrm{kabo} / \rightarrow$ [kaßo]. En fonología lo que conecta las representaciones fonológicas (representadas entre barras) y las representaciones fonéticas (representadas entre corchetes) son los procesos (la flecha entre /kabo/ y [kaßo]), los cuales son formalizados a través de reglas o constricciones.

Esta investigación no estudia las representaciones fonológicas (mentales) de los niños, por eso no usaremos el término "proceso" para explicar las divergencias que se observan entre el habla adulta y la de los niños.

En el presente trabajo las transcripciones del habla del modelo adulto son representadas entre barras (/glo.bo/), mientras que las transcripciones del habla infantil son representadas entre corchetes ([go.bo]).

Cuadro 2

Comparación del habla del modelo adulto y el de los niños

\begin{tabular}{cc} 
Modelo adulto & Producción del niño \\
/ glo.bo / & [go.bo ] \\
\hline
\end{tabular}


Ambas formas —/glo.bo/ y [go.bo] - correspondientes a la palabra "globo", deben ser entendidas como transcripciones fonéticas. La primera correspondiente al modelo adulto y la segunda al habla del niño.

En el ejemplo, se observa un tipo de divergencia entre la producción adulta y la del niño, que afecta la estructura silábica. Mientras en el modelo adulto la palabra tiene un grupo consonántico en la primera sílaba (es decir, /gl/); en la producción del niño, el grupo consonántico ha sido simplificado mediante la elisión de la consonante [1].

Preferimos el término 'divergencia' a 'error': Las diferencias observadas entre el modelo adulto y el habla de los niños no son errores involuntarios o asistemáticos cometidos por los niños que no pueden imitar correctamente el habla adulta, sino divergencias que reflejan las diferencias entre dos estados diferentes de conocimiento fonológico: un conocimiento ya maduro, en el caso del modelo adulto, y otro que está en proceso de madurar, en el caso del habla del niño.

A pesar de las diferencias, ambos tipos de conocimiento siguen reglas, principios o restricciones propios de la fonología que hacen que su estudio sea sistemático. Por este motivo, usaremos el término 'divergencia', el cual es más neutral y está libre de connotaciones asociadas con el término 'error' que no se aplica en el caso del habla de los niños. En este estudio, como ya hemos señalado, nos concentraremos en las divergencias que afectan la estructura de la sílaba (figuras 2 y 3 ).

El estudio de las divergencias en las estructuras silábicas entre el modelo adulto y el habla de los niños consiste en determinar qué características del modelo adulto son sustituidas o elididas en el habla del niño.

\section{La sílaba y su estructura}

Siguiendo los estudios hechos en el marco de la fonología autosegmental (Clements \& Keyser, 1983; Goldsmith, 1990; Kenstowicz, 1994; Liberman \& Prince, 1977), se asume que las representaciones fonéticas del habla adulta como las representaciones fonéticas del habla de los niños tienen una organización en niveles jerárquicos: el nivel del segmento, el nivel de la sílaba, el nivel de la palabra prosódica y el nivel de la frase fonológica. En el presente trabajo solo se involucra el nivel de la sílaba.

La sílaba es un constituyente fonológico que agrupa jerárquicamente segmentos (es decir, consonantes y vocales). La figura 1 muestra los segmentos consonánticos del castellano clasificados mediante rasgos fonológicos (Chomsky \& Halle, 1968; Clements, 1981, 1985, 1987, 1992; Halle, 1989, 1991, 1995; Halle, Vaux \& Wolfe, 2000). 
Figura 1

Clasificación de los segmentos consonánticos del modelo adulto

\begin{tabular}{|c|c|c|c|c|c|c|}
\hline & \multirow{2}{*}{ Labial } & \multicolumn{2}{|c|}{ Coronal } & \multirow{2}{*}{ Dorsal } & \\
\hline & & & [+anterior] & [-anterior] & & \\
\hline \multirow{2}{*}{\multicolumn{2}{|c|}{ [-continuo] }} & $p$ & $\mathrm{t}$ & & $\mathrm{k}$ & [-sonoro] \\
\hline & & $b$ & $d$ & & $g$ & [+sonoro] \\
\hline \multirow{2}{*}{\multicolumn{2}{|c|}{ [+continuo] }} & $f$ & $\mathrm{~s}$ & & $x^{\text {(José) }}$ & [-sonoro] \\
\hline & & & & $\mathrm{J}^{\wedge(\text { pollo })}$ & & [+sonoro] \\
\hline \multicolumn{2}{|c|}{ [africado] } & & & ch & & [-sonoro] \\
\hline \multicolumn{2}{|c|}{ [nasal] } & $\mathrm{m}$ & $\mathrm{n}$ & $\tilde{n}$ & & \\
\hline \multicolumn{2}{|c|}{ [-vibrante] } & & I & & & \\
\hline [+vibrante] & $\begin{array}{l}\text { [-tenso] } \\
\text { [+tenso] }\end{array}$ & & $\begin{array}{l}\mathrm{r}^{\text {(perro) }} \\
\mathrm{rr}(\text { perro) }\end{array}$ & & & \\
\hline
\end{tabular}

Nota: Aunque el Alfabeto Fonético Internacional (AFI) usa los símbolos [č] y [r] para representar la consonante africada sorda y la vibranre múltiple, respectivamente, nosotros hemos optado, por razones prácticas, por representarlas como $[\mathrm{ch}]$ y $[\mathrm{rr}]$.

La mayoría de las lenguas poseen estructura silábica formada por un "núcleo" (la vocal), que es obligatorio, y por una serie de sonidos consonánticos opcionales que aparecen tanto en el margen anterior al núcleo como en el margen posterior.

Pensemos, por ejemplo, en la palabra 'nariz'. Todo hablante nativo de castellano tiene intuiciones que le indican que esta palabra consta de dos sílabas /na/ y /ris/. El núcleo de la primera sílaba, / na /, es la vocal / a / y el núcleo de / ris / es / i /.
En castellano, la mayoría de las sílabas tiene una consonante inicial (a la cual le llamaremos simplemente "Inicio de Sílaba" o "Ataque"). En el caso de la sílaba / na /, su inicio es la consonante / $\mathrm{n} /$, y en el caso de / ris / es / $\mathrm{r} /$ (figura 2). El inicio de una sílaba en castellano puede estar formado por un grupo consonántico (como en la palabra 'flor', cuyo inicio tiene el grupo consonántico /fl/) o puede que la sílaba no tenga inicio; es decir, que el inicio esté vacío (como en la primera sílaba de la palabra 'uña'). 
Figura 2

El inicio de la sílaba

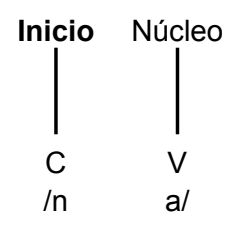

En castellano muchas sílabas poseen una consonante final (a la cual llamaremos "final de la sílaba" o "coda"). En el caso de la palabra 'nariz', la primera sílaba no tiene una consonante final. En contraste, la segunda sílaba, /ris/, sí la tiene: la consonante /s/. Finalmente, todas estas partes se unen y forman la unidad fonológica llamada "sílaba", que usualmente se representa por el símbolo ' $\sigma$ ' (figura 3 ).
Figura 3

La sílaba y sus partes
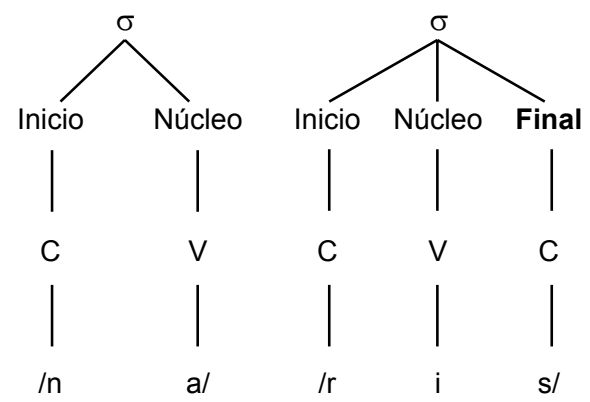

Tipos de divergencias relacionadas con la estructura silábica

Se entiende por divergencia de estructura silábica cuando, en comparación con el modelo adulto, el habla del niño muestra sílabas que han perdido/ganado segmentos o palabras en las cuales el orden de las sílabas ha cambiado (cuadro 3).

\section{Cuadro 3}

Ejemplos de divergencias relacionadas con la estructuración silábica

Modelo adulto
/cho.ko.la.te/ 'chocolate'
/bes.ti.ðo/ 'vestido'
/sa.pa.to/ 'zapato'
/glo.bo/ 'globo'
/rre.ga.lo/ 'regalo'
/gato/ 'gato'
/pje/ 'pie'
/cho.ko.la.te/ 'chocolate'

/cho.ko.la.te/ 'chocolate'

Habla del niño
[cho.ko.a.te]
[be.ti.ðo]
[pa.to]
[lo.bo]
[rre.ðe.ga.lo]
[gwato]
[pe]
[ko.cho.la.te]

Divergencias de estructura silábica

Elisión de inicio de sílaba

Elisión de final de sílaba

Elisión de sílaba átona

Reducción de grupo consonántico

Epéntesis

Diptongación

Monoptongación

Metátesis 
Comparado con el modelo adulto, los cambios en la estructura silábica observados en el habla de los niños tienden a simplificar sílabas complejas (es decir, sílabas con inicios, finales o núcleos complejos). El resultado de esta simplificación es una alta ocurrencia de sílabas formadas por una consonante seguida de una vocal, la estructura silábica universal.

En este estudio nos limitamos a analizar los siguientes tipos de divergencias relacionadas con la estructura silábica: elisión (omisión de segmentos), epéntesis (inserción de segmentos), metátesis (reordenamiento de segmentos), diptongación (cuando una vocal se convierte en un diptongo) y monoptongación (cuando un diptongo se convierte en una sola vocal) (cuadro 3).

\section{Método}

La presente es una investigación descriptiva, con un diseño no experimental y transversal. Se orientó fundamentalmente a explorar en qué estructuras silábicas los niños de 2,6 años a 4,11 años hablantes de castellano y que viven en la ciudad de Lima difieren con respecto a la producción del modelo adulto.

\section{Participantes}

Dadas las características de la investigación se optó por un muestreo no probabilístico de tipo intencional. Se incluyeron participantes de ambos sexos, entre 2,6 y 4,11 años (periodo sensible para observar divergencias relacionadas con la estructura silábica). Los niños asistían a nueve centros de educación inicial privados en los distritos de San Borja, Miraflores, Surco y La Molina, distritos pertenecientes a los niveles socioeconómicos A y B. ${ }^{1}$ No se incluyeron los niveles socioeconómicos C, D y E con la intención de controlar algunas variables sociales, como la restringida estimulación del lenguaje, la desnutrición, entre otros factores. Se siguieron los criterios de inclusión que a continuación se indican:

a. Niños de ambos sexos, pertenecientes a centros de educación inicial particulares mixtos de los niveles socioeconómicos $\mathrm{A}$ y $\mathrm{B}$.

b. Niños de edades comprendidas entre los dos años y medio y los cinco años.

c. Niños sin limitaciones intelectuales aparentes (según apreciación de los profesores de los niños seleccionados).

1 Los niveles socioeconómicos escogidos se encuentran definidos en base al análisis de las condiciones económicas y sociales de los jefes del hogar. A diferencia de los niveles C, D y E los hogares de los niveles A y B se caracterizan por tener jefes de hogares con un nivel de educación superior y con un promedio de estudio de entre 18 y 16 años, respectivamente, además de disfrutar de una comodidad económica estable (véase Informe general de marketing: Niveles socioeconómicos de la Gran Lima. Lima: Apoyo, Opinión y Mercado, octubre del 2006 [en línea]. $<$ www.apoyo.com/acerca/default.asp $>$. 
Tabla 1

Frecuencia de la muestra y porcentajes según los grupos de edad

\begin{tabular}{lrr}
\hline Edades & $\mathbf{F}$ & \% \\
\hline $2,6-2,11$ & 31 & 17.61 \\
$3,0-3,5$ & 35 & 19.88 \\
$3,6-3,11$ & 50 & 28.41 \\
$4,0-4,5$ & 30 & 17.05 \\
$4,6-4,11$ & 30 & 17.05 \\
Total & 176 & 100 \\
\hline
\end{tabular}

d. Niños sin pérdida auditiva aparente (según apreciación de los profesores de los niños seleccionados).

Como se observa en la tabla 1, fueron seleccionados 176 niños, 31 de ellos pertenecientes al grupo 1 , con edades comprendidas entre 2,6 y 2,11 años (el 17,61\% de la muestra total), 35 pertenecientes al grupo 2 , cuyas edades estuvieron entre 3,0 y 3,5 años (el $19,88 \%$ de la muestra total), 50 al grupo 3 de niños con edades entre 3,6 y 3,11 años (el 28,41\% de la muestra total), 30 al grupo 4 formado por alum- nos entre 4,0 y 4,5 años (el $17,05 \%$ de la muestra total), también 30 al grupo 5 de niños entre 4,6 y 4,11 años (el $17,05 \%$ de la muestra total).

\section{Técnica e instrumentos de recolección de datos}

Para los propósitos del estudio se elaboró un instrumento ad hoc de indagación del desarrollo fonológico. El instrumento estuvo compuesto por cuarenta y siete láminas de tamaño A4 que incluyeron dibujos sencillos a color, correspondientes a cuarenta y siete palabras.

\section{Cuadro 4}

Palabras presentadas en la recolección de datos

\begin{tabular}{|llllllllll}
\hline perro & gato & cocodrilo & doctor & dedo & uña & Nariz & boca & pie \\
ombligo & lengua & vestido & guante & calzón & media & zapato & collar & cuatro \\
manzana & chocolate & caramelo & agua & fresa & huevo & balde & columpio & triciclo \\
globo & cuento & libro & crayola & regalo & escoba & peine & cuchara & llave \\
árbol & jaula & negro & bandera & teléfono & foco & plancha & flor & algodón \\
espejo & blanco & & & & & & & \\
\hline
\end{tabular}


Las palabras presentadas fueron cuarenta y cuatro sustantivos, un adjetivo numeral y dos adjetivos calificativos de uso frecuente en el vocabulario infantil del habla de Lima (véase cuadro 4).

Las cuarenta y siete palabras presentaron una estructura silábica variada, considerando todos los segmentos consonánticos en diferentes posiciones dentro de las sílabas, con el propósito de observar la producción del niño en diferentes contextos. Se incluyeron dos palabras monosilábicas, veintiocho bisilábicas, trece trisilábicas y cuatro tetrasilábicas (tabla 2).

Con relación a la sílaba acentuada, hubo cuatro palabras agudas bisilábicas, una aguda trisilábica, veinticuatro graves bisilábicas, doce graves trisilábicas, tres graves de cuatro sílabas, una esdrújula de cuatro sílabas y dos monosilábicas.

De las cuarenta y siete palabras presentadas, trece están compuestas por sílabas con consonante final, trece por sílabas formadas por grupos consonán- ticos y once por sílabas con diptongos (cuadro 4).

\section{Procedimiento de recolección de datos}

Se recolectaron los datos de los niños que cumplían con los criterios de inclusión señalados. La recolección se hizo de manera individual, dentro del mismo centro educativo, con un tiempo de aplicación promedio de veinte minutos por alumno.

La consigna al momento de presentar la lámina fue: "Te voy a enseñar una figura. Dime cómo se llama". Cuando no se obtuvo una respuesta o que esta no correspondiera a la figura mostrada, se brindó información adicional sobre la lámina, y en reducidas ocasiones se requirió nominarlas, esperando la imitación. Es importante resaltar que el objetivo estuvo centrado en cómo el niño producía o decía las palabras y no en el reconocimiento de la lámina.

La producción fonética fue transcrita en una matriz de análisis (protocolo

Tabla 2

Número de sílabas y clasificación según acento de las palabras seleccionadas

\begin{tabular}{lccccc}
\hline \multirow{2}{*}{ Acentuación } & \multicolumn{5}{c}{ Número de sílabas } \\
\cline { 2 - 6 } & Monosílabas & Bisílabas & Trisílabas & Cuatrisílabas & Total \\
\hline Agudas & 2 & 4 & 1 & 0 & 7 \\
Graves & 0 & 24 & 12 & 3 & 39 \\
Esdrújulas & 0 & 0 & 0 & 1 & 1 \\
Total & 2 & 28 & 13 & 4 & 47 \\
\hline
\end{tabular}


de respuestas y análisis). Luego se calculó el número total de divergencias existentes por cada niño y posteriormente se analizaron, según los grupos de edad a través de la correlación de Pearson.

\section{ANÁLISIS Y DISCUSIÓN DE LOS RESULTADOS}

\section{Descripción de las divergencias relacionadas con la estructura silábica}

Basado en los datos recogidos, el análisis de las divergencias relacionadas con la estructura silábica muestra un mayor número de ocurrencias en el grupo de niños entre los 2,6 y 2,11 años y un menor número en el grupo de niños entre los 4,6 y 4,11 años (tabla 3).
En todos los casos, excepto la epéntesis (véase la columna con trama oblicua izquierda en la tabla 4), se observan diferencias significativas entre las medias del grupo $1(2,6$ y 2,11$)$ y las del grupo 5 (4,6 y 4,11). Las divergencias relacionadas con la estructura silábica disminuyen con la edad, es decir, a mayor edad, menor cantidad de divergencias.

Aunque las divergencias van disminuyendo con la edad, si comparamos las medias entre los grupos correlativamente ordenados (columnas sombreadas en la tabla 4) se puede apreciar que las diferencias son significativas solo entre el grupo $1(2,6$ y 2,11$)$ y el grupo $2(3,0$ y 3,5$)$ en todas las divergencias estudiadas: elisión de inicio de sílaba $(\mathrm{M}=2,97$ y D.S. $=3,37 ; \mathrm{M}=.91 \mathrm{y}$

Tabla 3

Media y desviación estándar de las divergencias de estructura silábica

\begin{tabular}{|c|c|c|c|c|c|c|c|c|c|c|}
\hline & \multicolumn{10}{|c|}{ Edades } \\
\hline & \multirow{2}{*}{\multicolumn{2}{|c|}{$\begin{array}{c}\text { Grupo } 1 \\
2,6-2,11\end{array}$}} & \multirow{2}{*}{\multicolumn{2}{|c|}{$\begin{array}{l}\text { Grupo } 2 \\
3,0-3,5\end{array}$}} & \multirow{2}{*}{\multicolumn{2}{|c|}{$\begin{array}{c}\text { Grupo } 3 \\
3,6-3,11\end{array}$}} & \multirow{2}{*}{\multicolumn{2}{|c|}{$\begin{array}{l}\text { Grupo } 4 \\
4,0-4,5\end{array}$}} & \multirow{2}{*}{\multicolumn{2}{|c|}{$\begin{array}{c}\text { Grupo } 5 \\
4,6-4,11\end{array}$}} \\
\hline & & & & & & & & & & \\
\hline & M & D.S. & M & D.S. & M & D.S. & M & D.S. & M & D.S. \\
\hline Elisión de inicio de sílaba & 2.97 & 3.37 & .91 & 2.42 & 0.27 & .45 & .06 & .25 & .03 & .18 \\
\hline Elisión de final de sílaba & 7.42 & 5.55 & 2.83 & 3.29 & .93 & 1.48 & .53 & .73 & .33 & .66 \\
\hline Reducción de grupo & 7.00 & 3.63 & 4.06 & 3.78 & 1.73 & 2.08 & 1.13 & 2.01 & 1.03 & 1.47 \\
\hline Elisión de sílaba átona & 1.03 & 1.99 & .20 & .58 & .00 & .00 & .10 & .31 & .17 & .38 \\
\hline Epéntesis & .55 & .81 & .26 & .61 & .17 & .47 & .30 & .53 & .20 & .66 \\
\hline Monoptongación & 2.19 & 2.82 & .46 & .92 & .23 & .57 & .03 & .18 & .06 & .25 \\
\hline Diptongación & .55 & 1.09 & .20 & .58 & .06 & .25 & .00 & .00 & .03 & .18 \\
\hline Metátesis & .71 & .86 & .29 & .52 & .20 & .48 & .20 & .55 & .03 & .18 \\
\hline
\end{tabular}


D.S. $=2,42)$, final de sílaba $(\mathrm{M}=7,42$; D.S. $=5.55$ y $\mathrm{M}=2,83$; D.S. $=3,29$ ), la reducción de grupo consonántico $(\mathrm{M}$ $=7$; D.S. $=3,63$ y $\mathrm{M}=4,06$; D.S. $=$ $3,78)$, la elisión de sílaba átona, $(\mathrm{M}=$ .03 ; D.S. $=1,99$ y $\mathrm{M}=.20$ : D.S. $=.58$ ) y la monoptongación $(\mathrm{M}=2,19 ;$ D.S. $=$ 2,82 y $\mathrm{M}=.46 ;$ D.S. $=.92$ ) excepto diptongación $(\mathrm{M}=0,55$; D.S. $=1,09 \mathrm{y}$ $\mathrm{M}=.20 ; \mathrm{D} . \mathrm{S} .=.58)$, epéntesis $(\mathrm{M}=$ .55 ; D. S. $=.81$ y M $=.26$; D.S. $=.61)$ y metátesis $(\mathrm{M}=.71 ; \mathrm{D} . \mathrm{S} .=.86$ y $\mathrm{M}=$ .29 ; D.S. = .52).

En el caso de la diptongación se observa una diferencia significativa entre el grupo $1(\mathrm{M}=0,55$; D.S. $=09)$ y el grupo $3(\mathrm{M}=.06$; D.S. $=.25)$. Lo mismo sucede en el caso de la metátesis grupo $1(\mathrm{M}=.71 ;$ D.S. $=.86)$ y el grupo $3(\mathrm{M}=.20 ; \mathrm{D} . \mathrm{S} .=.48)$. En el caso de la epéntesis, no existen diferencias significativas entre las medias de ninguno de los grupos de edad de nuestro estudio (tabla 4).

En lo referente a la reducción de grupos consonánticos, encontramos también diferencias significativas entre los grupos 2 y 3 (tabla 4 ).

\section{Baremos}

Basados en los resultados obtenidos, entregamos los resultados en "baremos", los cuales señalan el rango de divergencias comunes entre el modelo adulto y el habla de los niños para las divergencias relacionadas con la estructura silábica (tabla 5) según los grupos de edad.

Tabla 4

Comparación de medias de las divergencias de estructura silábica

\begin{tabular}{|c|c|c|c|c|c|c|c|c|c|c|}
\hline & \multicolumn{10}{|c|}{ Grupos de edades comparados } \\
\hline & $1-2$ & $1-3$ & $1-4$ & $1-5$ & $2-3$ & $2-4$ & $2-5$ & $3-4$ & $3-5$ & 4-5 \\
\hline $\begin{array}{l}\text { Elisión de inicio de } \\
\text { sílaba }\end{array}$ & $2.05^{*}$ & $2.70^{*}$ & $2.90^{*}$ & 2.93 & .65 & .85 & .88 & .20 & .23 & .03 \\
\hline $\begin{array}{l}\text { Elisión de final } \\
\text { de sílaba }\end{array}$ & $4.59^{*}$ & $6.49^{*}$ & $6.89^{*}$ & $7.09 *$ & 1.90 & 2.30 & $2.50^{*}$ & .40 & .60 & .20 \\
\hline $\begin{array}{l}\text { Reducción de grupo } \\
\text { consonántico }\end{array}$ & $2.94^{*}$ & $5.27^{\star}$ & $5.87^{*}$ & $5.97 \times$ & $2.32^{*}$ & $2.92^{*}$ & $3.02^{*}$ & .20 & .43 & .01 \\
\hline $\begin{array}{l}\text { Elisión de sílaba } \\
\text { átona }\end{array}$ & $1.83^{*}$ & $1.03^{*}$ & $.93^{*}$ & 87 & .20 & .10 & .03 & .10 & .17 & .06 \\
\hline Epéntesis & .29 & .38 & .25 & 0.35 & .09 & .04 & .05 & .13 & .03 & .10 \\
\hline Monoptongación & $1.74^{*}$ & $1.96^{*}$ & $2.16^{*}$ & $2.13 \%$ & .22 & .32 & .49 & .20 & .17 & .03 \\
\hline Diptongación & .35 & $.48^{*}$ & $.55^{\star}$ & $.52 \times$ & .13 & .20 & .17 & .06 & .03 & .03 \\
\hline Metátesis & .42 & $.51^{*}$ & $.51^{*}$ & $.68 *$ & .08 & .08 & .25 & .00 & .17 & .17 \\
\hline
\end{tabular}


Tabla 5

Baremo: Rango de divergencias de estructura silábica comunes por edades

\begin{tabular}{lcccccccccc}
\hline & \multicolumn{1}{c}{ Edades } \\
\cline { 2 - 11 } & $\mathbf{2 , 6}-\mathbf{2 , 1 1}$ & $\mathbf{3 , 0} \mathbf{- 3 , 5}$ & $\mathbf{3 , 6 - 3 , 1 1}$ & $\mathbf{4 , 0}-\mathbf{4 , 5}$ & $\mathbf{4 , 6 - 4 , 1 1}$ \\
\hline Elisión en inicio de sílaba & 0 & 6 & 0 & 3 & 0 & 1 & 0 & 0 & 0 & 0 \\
Elisión en final de sílaba & 2 & 13 & 0 & 6 & 0 & 2 & 0 & 1 & 0 & 1 \\
Reducción de grupo & 3 & 11 & 0 & 8 & 0 & 4 & 0 & 3 & 0 & 2 \\
Elisión de sílaba átona & 0 & 3 & 0 & 1 & 0 & 0 & 0 & 0 & 0 & 1 \\
Epéntesis & 0 & 1 & 0 & 1 & 0 & 1 & 0 & 1 & 0 & 1 \\
Monoptongación & 0 & 5 & 0 & 1 & 0 & 1 & 0 & 0 & 0 & 0 \\
Diptongación & 0 & 2 & 0 & 1 & 0 & 0 & 0 & 0 & 0 & 0 \\
Metátesis & 0 & 2 & 0 & 1 & 0 & 1 & 0 & 1 & 0 & 0 \\
\hline
\end{tabular}

\section{CONCLUSIONES}

La importancia de estos resultados radica en la identificación de qué tipo de divergencias en la estructura silábica ocurren en el habla infantil cuando se le compara con el modelo adulto y hasta cuándo se puede esperar que tales divergencias subsistan en el habla de los niños.

En el grupo de investigación se encontraron varias clases de divergencias relacionadas con la estructura silábica: elisión de segmento en inicio y final de sílaba, reducción de grupo consonántico, elisión de sílaba átona, epéntesis, monoptongación, diptongación y metátesis.

Como era de esperarse, estas divergencias disminuyeron con la edad en la mayoría de los casos; así, a partir de los
4,6 años se encuentra que la posibilidad de ocurrencia de una divergencia no supera el 1,0 y que solo en la reducción de grupos consonánticos se encuentra una media de 1.03

El periodo comprendido entre los 2,11 y 3,5 años es el más sensible, encontrándose diferencias significativas entre ambas edades en elisión de inicio y final de sílaba, reducción de grupo consonántico, elisión de sílaba átona y monoptongación.

En el caso de la diptongación y la metátesis el decremento importante es en el periodo comprendido entre los 3,6 y 3,11 años. En el caso de la epéntesis hay poca variabilidad entre los grupos estudiados.

En cuanto a la reducción de grupos consonánticos hay dos momentos de 
decremento significativo: el primero, ya reportado, y el segundo a partir de los tres años y medio. Esto es, se mantiene una disminución significativa de la reducción de grupos consonánticos entre los 2,6 y 3,5 años.

La reducción de grupos consonánticos y la elisión de final de sílabas son las divergencias más frecuentes que encontramos en este grupo de edades, lo cual es muy significativo en tanto que Hodson (1986) sostiene que estas dos divergencias, más la omisión de sílabas en la estructura silábica, determinan la diferencia entre un habla inteligible y una no inteligible a los oyentes no cercanos al niño hablante.

Nuestros resultados señalan que el periodo más sensible para la adquisición de los patrones silábicos del castellano, donde disminuyen significativamente las divergencias entre el habla del modelo adulto y la de los niños es entre $\operatorname{los} 2,6$ y 3,6 años. Este es señalado como un periodo sensible, con todas las connotaciones que esto implica para la estimulación oportuna, en especial de la familia, y sus repercusiones en el proceso de desarrollo de la función comunicativa.

Los resultados no solo corroboran los hallazgos reportados en otros estudios, que demuestran que el sistema fonológico se va adquiriendo de manera progresiva y que el niño utiliza diferentes estrategias para aproximarse a los sonidos usados por los adultos pero también representan el primer estudio detallado del desarrollo de la adquisición de la sílaba en el castellano hablado por niños en Lima.

Este estudio nos permitió la elaboración de baremos, con las limitaciones que la muestra implica, pero que puede brindar un punto de referencia a los psicólogos, maestros, terapeutas del lenguaje, entre otros profesionales interesados en el desarrollo del lenguaje, para determinar si las divergencias observadas en los niños a una cierta edad son parte del desarrollo fonológico normal o, en caso contrario, es necesario aplicar a tiempo la estimulación necesaria.

\section{REFERENCIAS}

Acosta, V. M. \& Moreno, A. M. (1999). Dificultades del lenguaje en ambientes educativos. Barcelona: Masson.

Acosta, V. M.; Ramos, V. \& León, S. (1998). Dificultades del habla infantil: Un enfoque clínico. Investigación teoría y práctica. Málaga: Aljibe.

Aguado, G. (1999). Trastorno especifico del lenguaje. Retraso de lenguaje y disfasia. Málaga: Aljibe.

Baertsch, K. (2002). An Optimalitytheoretic approach to syllable structure: The Split Margin Hierarchy. Ph.D. Dissertation, Indiana University, Bloomington. 
Blevins, J. (1995). The syllable in phonological theory. En J. A. Goldsmith (Ed.). The handbook of phonological theory (pp. 206-244). Cambridge, Mass., y Oxford, UK: Blackwell.

Bloom, L. \& Lahey, M. (1978). Language development and language disorders. Nueva York: John Wiley and Sons.

Bosch, L. (1982). Prueba para la evaluación del desarrollo fonológico en niños de tres a siete años y once años. Tesis. Universidad de Barcelona.

Bosch, L. (1984). El desarrollo fonológico infantil: Una prueba para su evaluación. Madrid: Pirámide.

Bosch, L. (2003). Trastornos del desarrollo fonético y fonológico. En M. Puyuelo \& J. A. Rondal (Ed.). Manual de desarrollo y alteraciones del lenguaje: Aspectos evolutivos. Nueva York: Wiley.

Bromberger, S. \& Halle, M. (1989). Why phonology is different. Linguistic Inquiry, 20, 51-70.

Broselow, E.; Chen, S.-I. \& Huffman, M. (1997). Syllable weight: Convergence of phonology and phonetics. Phonology, 14, 47-82.

Cairns, C. E. \& Feinstein, M. H. (1982). Markedness and the theory of syllable structure. Linguistic Inquiry, 13(2), 193-226.
Ciccia, C.; Montezuma, O.; ElíasUlloa, J.; Gabillo, G.; Echenique, M.; Raffo, G.; Seminario. A. (2006). Las divergencias fonológicas entre el habla adulta y el de los niños en el español hablado en Lima Perú. Revista de Logopedia, Foniatría y Audiología, vol. 24, 4.

Chomsky, N. \& Halle, M. (1968). The sound pattern of English. Nueva York: Harper \& Row.

Clements, G. N. (1981). The hierarchical representation of tone features. En I. R. Dihoff (Ed.). Current approaches to African linguistics. Vol. 1, pp. 145-176. Dordrecht: Foris.

Clements, G. N. (1985). The geometry of phonological features. Phonology Yearbook, 2, 225-252.

Clements, G. N. (1987). Phonological feature representation and the description of intrusive stops. En A. Bosch, B. Need \& E. Schiller (Eds.), CLS 23: Parasession on Autosegmental and Metrical Phonology (pp. 29-50). Chicago: Chicago Linguistic Society.

Clements, G. N. (1992). Phonological primes: Features or gestures? Phonetica, 49, 181-193.

Clements, G. N., \& Keyser, S. J. (1983). CV Phonology: A generative theory of the syllable. Cambridge, Mass.: MIT Press. 
Goldsmith, J. (1990). Autosegmental and metrical phonology. Oxford \& Cambridge, Mass.: Blackwell.

Halle, M. (1989). The intrinsic structure of speech sounds. Cambridge, Mass.: MIT.

Halle, M. (1991). Phonological features. En W. Bright (Ed.). Oxford International Encyclopedia of Linguistics (pp. 207-212. Nueva York: Oxford University Press.

Halle, M. (1995). Feature geometry and feature spreading. Linguistic Inquiry, 26, 1-46.

Halle, M. (2002). From memory to speech and back: Papers on phonetics and phonology, 1954-2002. Berlín / Nueva York: Mouton de Gruyter.

Halle, M., \& Idsardi, W. (1995). General properties of stress and metrical structure. En J. A. Goldsmith (Ed.). The Handbook of Phonological Theory (pp. 403-443). Cambridge, Mass., y Oxford, UK: Blackwell.

Halle, M.; Vaux, B. \& Wolfe, A. (2000). On feature spreading and the representation of place of articulation. Linguistic Inquiry, 31, 387-444.

Harris, J. (1983). Syllable structure and stress in Spanish: a nonlinear analysis. Cambridge, Mass.: MIT Press.

Harris, J. W. (1989). Our present understanding of Spanish syllable structure. En P. Bjarkman \& R.
Hammond (Eds.). American Spanish Pronunciation (pp. 151169). Washington, DC: Georgetown University Press.

Hironymous, P. (1999). Selection of the optimal syllable in an alignmentbased theory of sonority. Unpublished Ph. D. Dissertation, University of Maryland, College Park, MD.

Hodson, B. W. \& Paden, E. P. (1991). Targeting intelligible speech: a phonological approach to remediation (2. ${ }^{\mathrm{a}}$ edición). Austin, Tex.: Pro-Ed.

Hodson, B. W. (1986). The assessment of phonological processes-Spanish. San Diego: Los Amigos Association.

Kenstowicz, M. (1994). Phonology in generative grammar. Oxford: Blackwell.

Lee, J. F. (1989). The acquisition of syllable structure and stress patterns by monolingual Spanish-speaking children. Hispanic Linguistics, 2, 229-252.

Lenneberg, E. H. (1981). Fundamentos biológicos del lenguaje. (2. ${ }^{\mathrm{a}}$ edición). Madrid: Alianza Universidad.

Levelt, C. C., Schiller, N. O. \& Levelt, W. J. M. (1999). A developmental grammar for syllable structure in the production of child langauge. Brain and Language, 68, 291-299. 
Liberman, M. \& Prince, A. (1977). On stress and linguistic rhythm. Linguistic Inquiry, 8, 249-336.

Narbona, J. (2006). El lenguaje del niño $\mathrm{y}$ sus trastornos. En $\mathrm{N}$.
Fejerman \& E. Fernández (Eds.). Neurología pediátrica. MadridBuenos Aires: Panamericana.

Richelle, M. (1984). La adquisición del lenguaje. Barcelona: Herder. 